\title{
A Technology for Multiscale Edge Estimation, Data Compression and Pattern Matching based on the Concept of Laplacian and Gaussian Pyramids
}

\author{
Archana R Priyadarshini \\ Asst. Professor, Dept. of CSE \\ CEC, Bantwal Taluk \\ Karnataka, India
}

\begin{abstract}
Image pyramid provides multi-resolutional format that mirrors multiple scales of processing in the human visual system. Pyramid construction tends to enhance image features, such as edges, which are important for interpretation. Gaussian pyramid techniques are efficiently used for multi-scale edge estimation, to compute coarse scale images and also for finer details of the images .Laplacian pyramids methodology help in efficient compression and redundancy removal. The methodologies that are into picture helps to develop filterbased representations in order to decompose images into information at multiple scales, to extract features of interest and also in attenuating noise.
\end{abstract}

\section{Keywords}

Multi-resolutional; coarse image; human visual system; compression and redundancy removal; bandpass

\section{INTRODUCTION}

Pyramid methods may be applied to analysis in several ways. The first concerns pattern matching, next to estimate the integrated properties within local image regions and also fast coarse fine search techniques.

Laplacian pyramid has been described as a data structure composed of bandpass copies of an image that is well suited for scaled-image analysis. But the pyramid may also be viewed as an image transformation. There are 2 reasons for transformation:

a) The transformation may isolate critical components of the image pattern so they are more directly accessible for analysis.

b) The transformation may place the data in more compact form so that thay can be stored and transmitted more efficiently.

Laplacian pyramid serves both of these objectives. This method also helps in creating high fidelity image from a set of images taken with different focal lengths. Gaussian pyramid serves to be useful in multiscale edge estimation and also for constructing large digital image from smaller subsamples of the image with the help of structural information.

\section{LAPLACIAN AND GAUSSIAN PYRAMID OVERVIEW}

The Gaussian pyramid is computed as follows. The original image is convolved with a Gaussian kernel. The resulting image is a low pass filtered version of the original image. The cut-off frequency can be controlled using the parameter $\boldsymbol{\sigma} \sigma$. The Laplacian is then computed as the difference between the original image and the low pass filtered image. This process is continued to obtain a set of band-pass filtered images (since each is the difference between two levels of the Gaussian pyramid). Thus the Laplacian pyramid[11](as seen in Figure1) is a set of band pass filters.

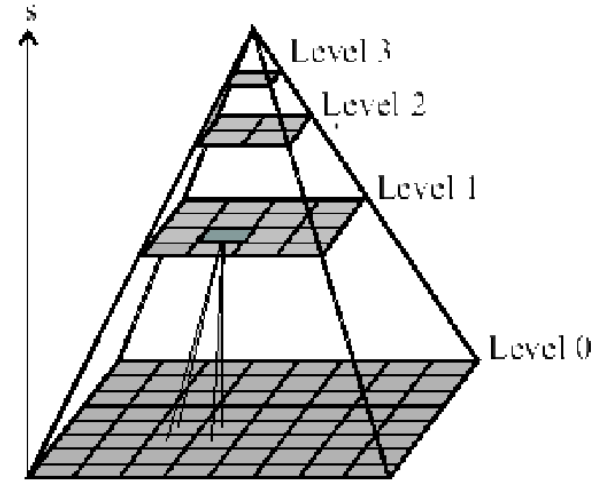

Fig 1:Laplacian Pyramid

.General Approach for Laplacian Pyramid blending[12]:

1. Build Laplacian pyramids $L A$ and $L B$ from images $A$ 2.1 and $B$

2. Build a Gaussian pyramid $G R$ from selected region $\boldsymbol{R}$

3. Form a combined pyramid $L S$ from $L A$ and $L B$ using nodes of

2.2 $G R$ as weights:

- $\quad L S(i, j)=G R(I, j) * L A,(I, j)+(1-G R(I, j)) * L B(I, j)$

4. Collapse the $L S$ pyramid to get the final blended image

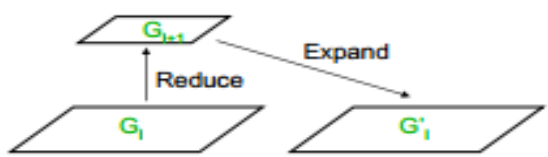

Fig 2: Gaussian Pyramid

Here G0,G1 are levels of Gaussian pyramid[13](as seen in Figure2). Predict level G1 from level G1 from level G1+1 by expanding G'1.

Denote by $\mathrm{Ll}$ the error in prediction:

$$
L_{1}=G_{1} \cdot G_{1}^{\prime}
$$

$\mathrm{L} 0, \mathrm{~L} 1, \ldots .=$ the levels of a Laplacian Pyramid 


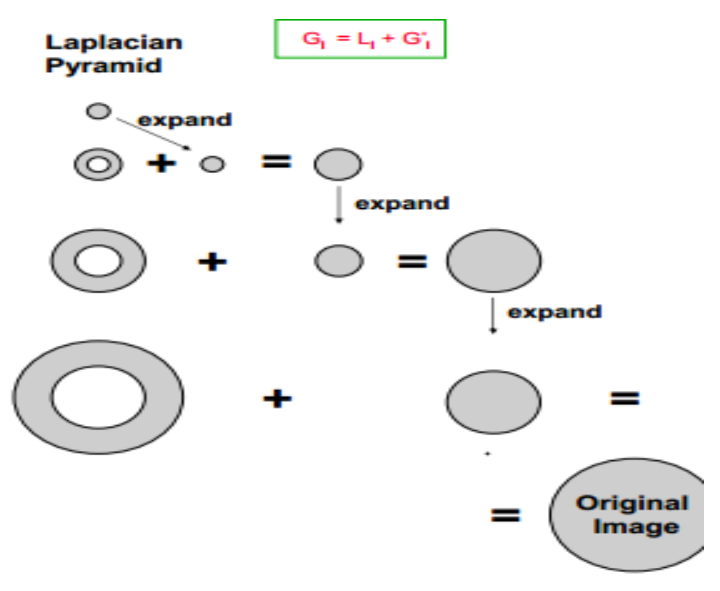

\subsection{Gaussian Pyramid Reconstruction}

The Gaussian pyramid construction[12](as shown in Figure3) applies a filter mask to the image until minimum resolution is reached.

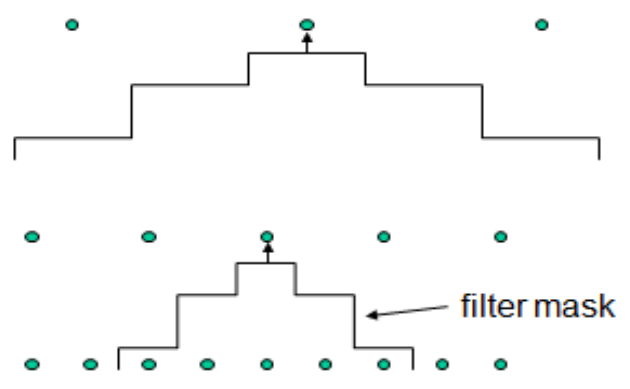

Fig 3:Gaussian pyramid construction

The pyramids based method that was applied to an image is as shown in Figure4.

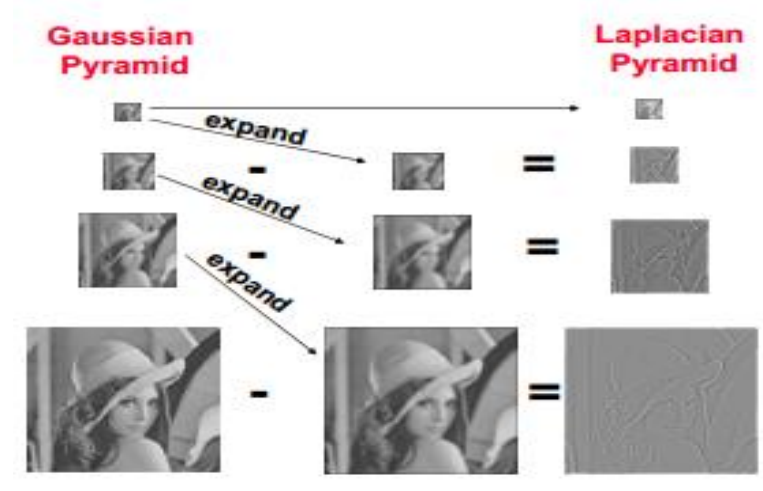

Fig 4: Gaussian versus laplacian

Gaussian pyramid is a sequence of low-pass, down-sampled images, $[\mathrm{I} 0, \mathrm{I} 1, \ldots \mathrm{IN}]$ where $\mathrm{I} 0, \mathrm{I} 1, \ldots \mathrm{IN}$ are all vectors.Usually constructed with a separable $1 \mathrm{D}$ kenel $\mathrm{h}=[\mathrm{h} 1 ; \mathrm{h} 2 ; \mathrm{h} 3 ; \mathrm{h} 4$; h5], and a down-sampling factor of 2 (in each Direction).

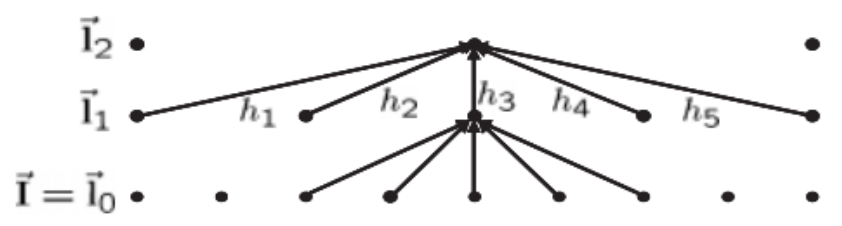

In matrix notation(1D), the mapping from one level to next has the form

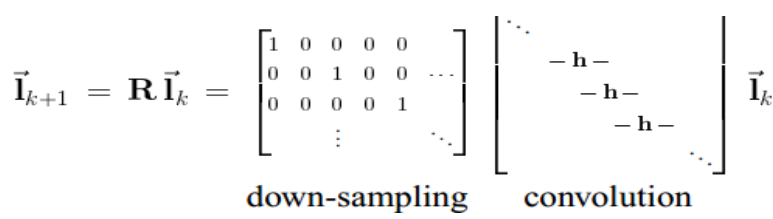

Laplacian pyramid: Overall decomposition is based on the difference of low-pass filters . Image is recursively decomposed into low-pass and highpass bands. Each band of Laplacian pyramid is the difference between two adjacent low-pass images of gaussian pyramid, $[\mathrm{I} 0, \mathrm{I} 1, \ldots \mathrm{IN}]$ where $\mathrm{I} 0, \mathrm{I} 1, \ldots . \mathrm{IN}$ are vectors. That is:

$$
\overrightarrow{\mathbf{b}}_{k}=\overrightarrow{\mathbf{l}}_{k}-\mathbf{E} \overrightarrow{\mathbf{l}}_{k+1}
$$

Here the second term is an up-sampled smoothed version of $\mathrm{IK}+1$ Vector.

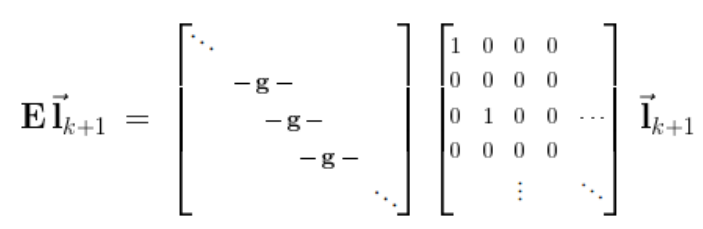

$$
\text { Convolution up-sampling }
$$

Construction of:

$$
\begin{aligned}
{\left[\overrightarrow{\mathbf{b}}_{0},\right.} & \left.\overrightarrow{\mathbf{b}}_{1}, \ldots, \overrightarrow{\mathbf{b}}_{L-1}, \overrightarrow{\mathbf{l}}_{L}\right] \\
\overrightarrow{\mathbf{l}}_{0} & =\overrightarrow{\mathbf{I}} \\
\overrightarrow{\mathbf{l}}_{k+1} & =\mathbf{R} \overrightarrow{\mathbf{l}}_{k} \\
\overrightarrow{\mathbf{b}}_{k} & =\overrightarrow{\mathbf{l}}_{k}-\mathbf{E} \overrightarrow{\mathbf{l}}_{k+1}
\end{aligned}
$$

Reconstruction of I vector is exact and straightforward.

$$
\begin{aligned}
\overrightarrow{\mathbf{l}}_{k} & \doteq \overrightarrow{\mathbf{b}}_{k}+\mathbf{E} \overrightarrow{\mathbf{l}}_{k+1} \\
\overrightarrow{\mathbf{I}} & =\overrightarrow{\mathbf{l}}_{0}
\end{aligned}
$$

Gaussian pyramid that was applied for the lenna image is as shown below in Figure5:

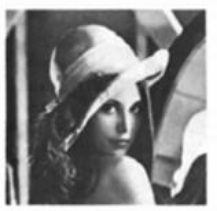

0

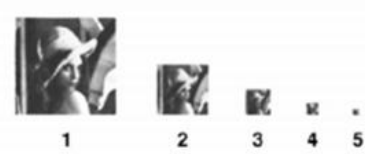

Fig 5: Gaussian pyramid
Laplacian pyramid(as shown in Figure6)[12] was applied to a lenna image. 


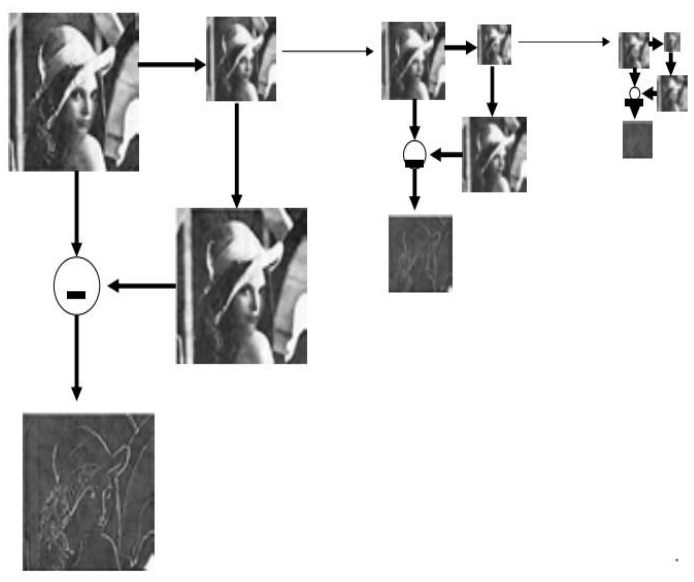

Fig 6: Laplacian Pyramid

\section{RESULT ANALYSIS}

Here 5grayscaled images are taken into consideration(as seen in Figure7, Figure8, Figure9,Figure10). Initially tests of images are taken.Image read operation is performed for all images. Next comes the preparation of kernels. Convolution of the input image with its kernel is done for each of the images taken(as seen in Figure12, Figure13, Figure14, Figure15, Figure16). It is done as follows: Given Im as a gray scale image. convolve(Im, Fil, str) This function performs convolution of gray scale image Im with the filter Fil.

$[\mathrm{ik}, \mathrm{iw}]=\operatorname{size}(\mathrm{Im})$;

$[\mathrm{fk}, \mathrm{fw}]=\operatorname{size}($ Fil $)$;

This is a two dimensional array of size iw by ik. Fil denotes a filter. This is a two dimensional array of floating point numbers of size fw by fk. Usually ( fk, fw ) $\ll(i k$, iw). The output $\mathrm{O}(\mathrm{x}, \mathrm{y})$ is an image of same size as $\mathrm{Im}$. The value of $\mathrm{O}$ at any pixel is generated by position Fil on $\operatorname{Im}(\mathrm{x}, \mathrm{y})$ such that the top right pixel of Fil coincides with $\operatorname{Im}(x, y)$ and then multiplying the values of Im and Fil for all the pixels of Im covered by Fil, and finally summing these values.Next procedure is to apply low pass filtering to the image. This includes firstly preparation of inputs and the kernel.i.e. the inputs include gray image and a Box filter $(2 \times 2)$. Then there are 8 levels of processing performed. Thus 8 level Gaussian pyramids are obtained using convolution (i.e.for each of the images). Here the few levels out of 8 levels of pyramid for the $1^{\text {st }}$ image are depicted in Figure18,Figure19,Figure20.Next is the application of bandpass filtering.This involves the generation of 7 levels of the Laplacian pyramid by subtracting the consecutive levels of gausssian pyramids. Here few levels that are visible are depicted in Figure21, Figure22, Figure23, Figure24, Figure25.Here bilinear interpolation to upsample the image of lower size is used so that the two images used during subtraction have the same size.Here 2 approaches were used for computing the Gaussian pyramid. First approach is by the use of Box filter where size of image gets reduced at each level. Second approach is zero crossings where the size of the image remains fixed and during convolution process results in black border around the lower and left periphery. These are seen in Figure26, Figure27, Figure28, Figure29, Figure30 and Figure31. Laplacian computation with equal size images are shown in Figure32,Figure33 and Figure34. Then multi-scale edge estimation(convolution,segmentation and zero crossing) is performed on the images. These are depicted in Figure35, Figure36 and finally Figure37.

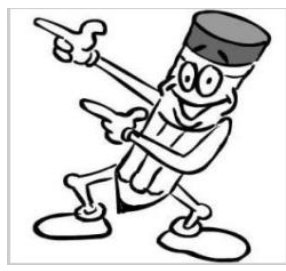

Fig 7 :Image1

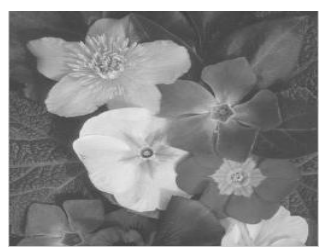

Fig 8: Image2

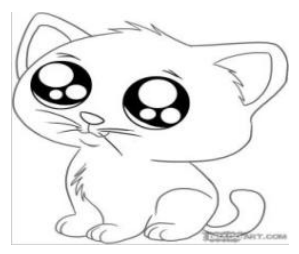

Fig 9 :Image3

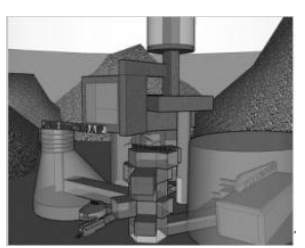

Fig 10:Image4

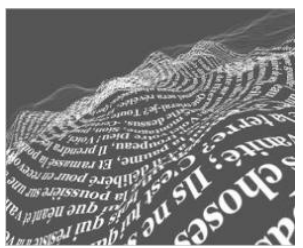

Fig 11: Image5

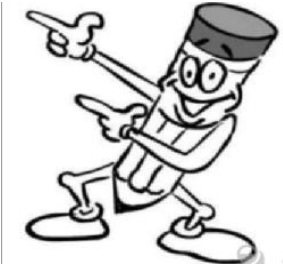

Fig 12 :Image6

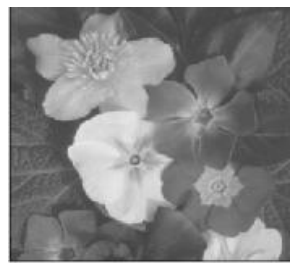

Fig 13:Image7 


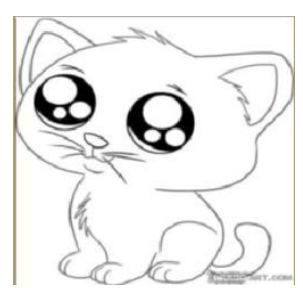

Fig 14:Image8

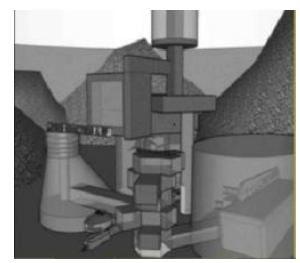

Fig 15:Image9

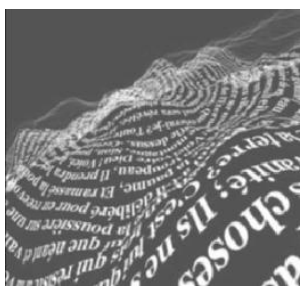

Figure 16:Image10

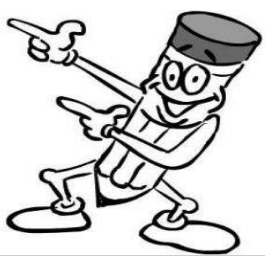

Fig 17:Image11

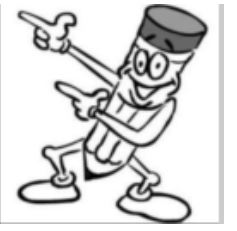

Fig 18:Image12

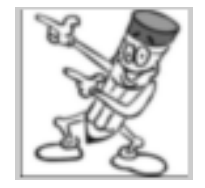

Fig 19:Image13

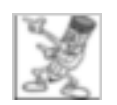

Fig 20:Image14

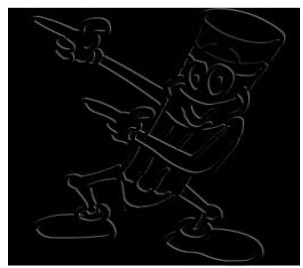

Fig 21:Image15

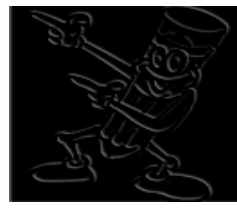

Fig 22:Image16

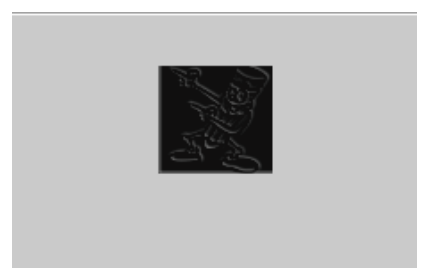

Fig 23:Image17

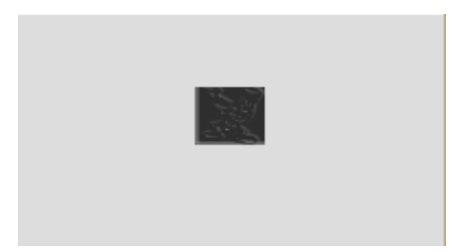

Fig 24:Image18

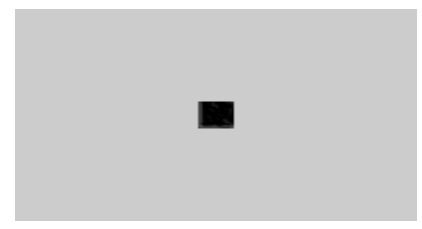

Fig 25:Image19

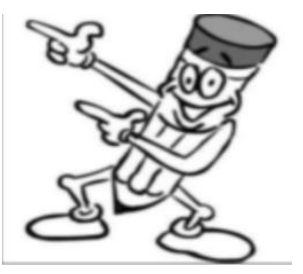

Fig 26:Image20

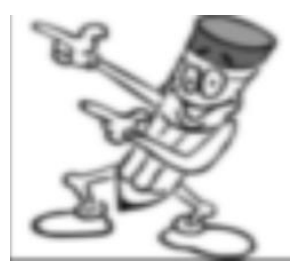

Fig 27:Image21

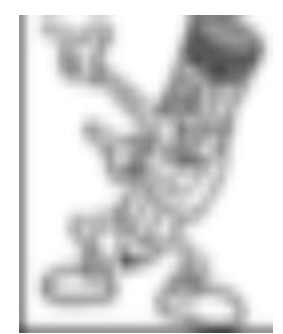

Fig 28:Image22 


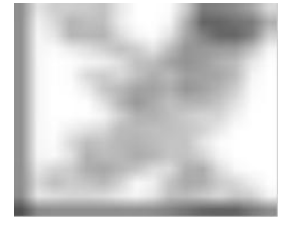

Fig 29:Image23

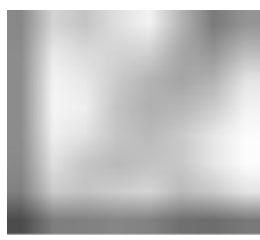

Fig30:Image24

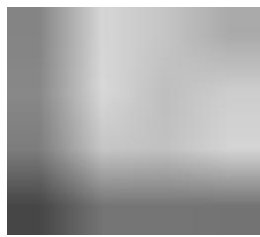

Fig31: Image25

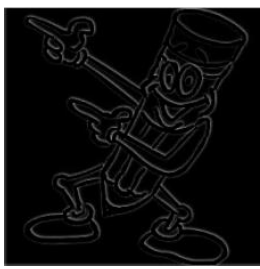

Fig32:Image26

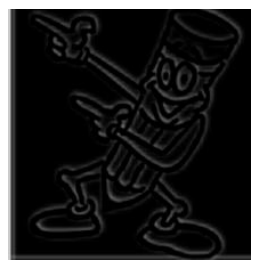

Fig 33:Image27

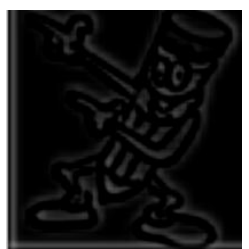

Fig 34:Image28

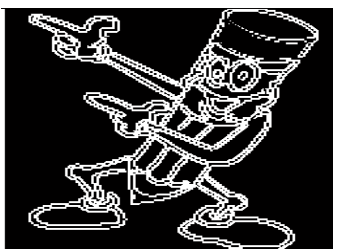

Fig 35:Image29

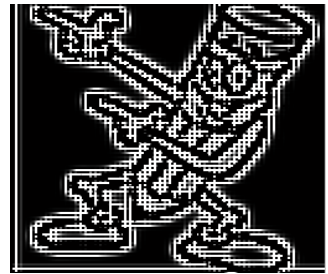

Fig36:Image30

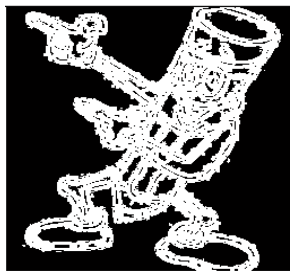

Fig 37:Image31

\section{CONCLUSIONS}

Gaussian pyramid results in progressively blurred and sub sampled versions of the image. Laplacian pyramid shows the information added in Gaussian pyramid at each spatial scale. This is helpul for noise reduction \& coding. Thus these methods have been effective in performing data compression, multi-scale edge estimation and also in extracting the target pattern for a set of images. As an extension of the work, different box-filter size could be used and also this work could be extended for color images.

\section{REFERENCES}

[1] Shibin $\mathrm{Wu}$, Shaode Yu, Yuhan Yang and Yaoqin Xie, "Feature and Contrast Enhancement of Mammographic Based on Multiscale Analysis and Morphology", Computational and Mathematical Methods in Medicine Volume 2013 (2013), Article ID 716948.

[2] Wencheng Wang and Faliang Chang ,"A Multi-focus Image Fusion Method Based on Laplacian Pyramid", JOURNAL OF COMPUTERS, VOL. 6, NO. 12 , DECEMBER 2011.

[3] S. Paris, S. W. Hasinoff, and J. Kautz, "Local Laplacian Filters: Edge-Aware Image Processing with a Laplacian Pyramid," ACM Transactions on Graphics (Proceedings of SIGGRAPH, Vol. 30, Issue 4, Article 68, 2011.

[4] P. Bhat, C. L. Zitnick, M. Cohen, and B. Curless, "Gradientshop: A Gradient-Domain Optimization Framework for Image and Video Filtering," ACM Transactions on Graphics, Vol. 29, Issue 2, Article 10, 2010.

[5] K. Subr, C. Soler, and F. Durand, "Edge-Preserving Multiscale Image Decomposition Based on Local Extrema," ACM Transactions on Graphics (Proc. SIGGRAPH Asia), Vol. 28, Issue 5, Article 147, 2009.

[6] S. Bae, S. Paris, and F. Durand, "Two-Scale Tone Management for Photographic Look," ACM Transactionsion Graphics (Proc. SIGGRAPH), Vol. 25, Issue 3, pp. 637-645. 2006.

[7] G. Aubert and P. Kornprobst, "Mathematical Problems in Image Processing: Partial Differential Equations and The Calculus of Variations," Applied Mathematical Sciences, Springer, Munich, Vol. 147,2002. 
International Journal of Computer Applications (0975 - 8887)

Volume 110 - No. 10, January 2015

[8] Mill Xbt, AMelhmid Hachicha. and blain Mtrigot ,"An Efficient Parallel Implementation of the Laplacian Pyramid Algorithm" MvA'92 1APR Worshop on MachineVision Applications Dec.7-9,1992, Tokyo.

[9] P. J. Burt and E. H. Adelson, "The Laplacian Pyramid as a Compact Image Code," IEEE Transactions on Communication, Vol. 31, Issue 4, pp. 532-540, 1983.
[10] http://www.cs.toronto.edu/ mangas/teaching/320/assign ments/a3/

[11] http://www.cs.utah.edu/ arul/report/node12

[12] http://graphics.cs.cmu.edu/courses/15463/2005_fall/ww w/Lectures/Pyramids

[13] http://cs.haifa.ac.il/hagit/courses/ip/Lectures/Ip11_Multis caleRepx4. 